\title{
METHODOLOGY OF EXPERIMENTAL INVESTIGATIONS OF VALVE OPERATION
}

\author{
Seyidahmadov Natig Sabir \\ Azerbaijan State Scientific Research Institute of Labor Protection and Technical Safety \\ 108 Tabriz str., Baku, Azerbaijan, AZ1008 \\ n.natiq.az@mail.ru
}

\begin{abstract}
Tightness, as well as the reliability of the valve plate, is a complex property of the effective operation of compressor cylinders of the first stage and, in general, gas-engine reciprocating compressors. The issue of valve plate tightness is a subject of independent study, since technical and economic efficiency depends on their work. In this connection, only some data obtained under operating conditions are presented in this work.

As a research result, it is found that, taking into account the identified requirements for the gas lift system, in order to effectively increase the operating hours of valves with increased tightness of the plate, it is necessary to check and purge the valves. Therefore, each valve in the gas lift compressor station, without subjecting them to cleaning, is first recommended to check for leaks. To confirm the feasibility of checking valve tightness, special equipment is offered for each gas-lift compressor station, a purge chamber, on which the tightness of valve plates is checked.

The usefulness and importance of the purge chamber is in preparation of the valve at the gas lift compressor station, which contributes to increased efficiency, safe operation, normal tightness and reliability of its operation.
\end{abstract}

Keywords: direct-flow valves, purge chamber, valve plates, tightness, oil, gas, gas lift operation, gas-engine piston compressor.

DOI: $10.21303 / 2461-4262.2019 .00991$

\section{Introduction}

It is known $[1,2]$ that the capacity of compressor equipment operated in gas lift and associated petroleum gas transportation systems is constantly increasing. The scope of its application is expanding, as a result of which it becomes very important to conduct experimental work at a gas lift compressor station to study the improvement of the efficiency and tightness of piston compressor valves. It is advisable to evaluate the effectiveness of reciprocating compressor equipment comprehensively, based on the results of a study and analysis of the main technological indicators of operation, which requires special attention.

An experimental evaluation of the efficiency and tightness of the valves of the compressor cylinders of the first stage of gas-engine reciprocating compressors is one of the obligatory steps in the process of checking, preparing and increasing the tightness. Therefore, the efficiency and safety of operation of the valves of the compressor unit. Therefore, the experimental study of valve tightness and increasing their operational efficiency, as well as the analysis of technological parameters before and after the experimental study, is very important [3, 4].

Regulatory, technical and methodological documentation for the operation of valves of reciprocating compressors, as a rule, are concise. These documents do not provide for unforeseen shortcomings during operation associated with the physicochemical properties of the booster associated petroleum gas. They also do not contain justifications and explanations of the established requirements. Without claiming to be an exhaustive exposition of all the questions of an experimental study of valve tightness and increasing their operational efficiency, let's highlight only some aspects of this important problem. Problems of maintaining the performance of a reciprocating compressor unit operating in gas lift and gas transport systems $[5,6]$.

\section{Research results of valves directly at the gas lift compressor station}

As a rule, in a gas lift compressor station, all valves are subject to external inspection before being installed on the compressor cylinders. Inspections should be recorded in the technical journal for each mining and metallurgical complex. If defects are found, they are eliminated, and then the valve is installed on the cylinder seat. If the valves have reached their service life, they must be replaced with new ones [7]. 
As experience shows, an external inspection directly by personnel at a gas lift compressor station does not completely detect defects such as plate breakage, seizure on the saddle, shells in the lift limiter, chipping of individual sections of the plate and saddle, or violation of the splinting of parts.

Considering that the largest number of failures is associated with valves of compressor cylinders of the first stage, an experimental assessment of their efficiency and tightness is necessary, which is one of the mandatory steps in the process of checking the safety of a compressor unit $[8,9]$.

In the process of experimental studies of direct-flow valves, the following formula was adopted as a criterion:

$$
t=t_{1} \frac{V}{V_{1}} \sqrt{\frac{T_{1}}{T}},
$$

where $t$ - valve density time; $t_{1}$ - time of change of overpressure in the vessel (minimum permissible value according to the manufacturer's instructions $t_{1}=40 \mathrm{~s}$ ); $V$ - volume of the test vessel, $\mathrm{m}^{3}$, $V=0.065 \mathrm{~m}^{3} ; V_{1}$ - actual volume of the test tank, $V=0.075 \mathrm{~m}^{3} ; T$ - standard gas temperature, $T=293.15 \mathrm{~K}$; $T_{1}$ - actual gas temperature, $T_{1}=299.15 \mathrm{~K}$.

Substituting all the values in the formula (1), let's obtain

$$
t=4 \frac{0,065}{0,075} \sqrt{\frac{299,15}{293,15}}=35 s .
$$

Thus, the set valve density time $t=35 \mathrm{~s}$ complies with manufacturer's recommendations.

The valves were examined directly at the gas-lift compressor station in order to determine their static characteristics in a purge chamber connected to the compressed air piping scheme. Scheme of the stand for static valve purge is shown in Fig. 1.

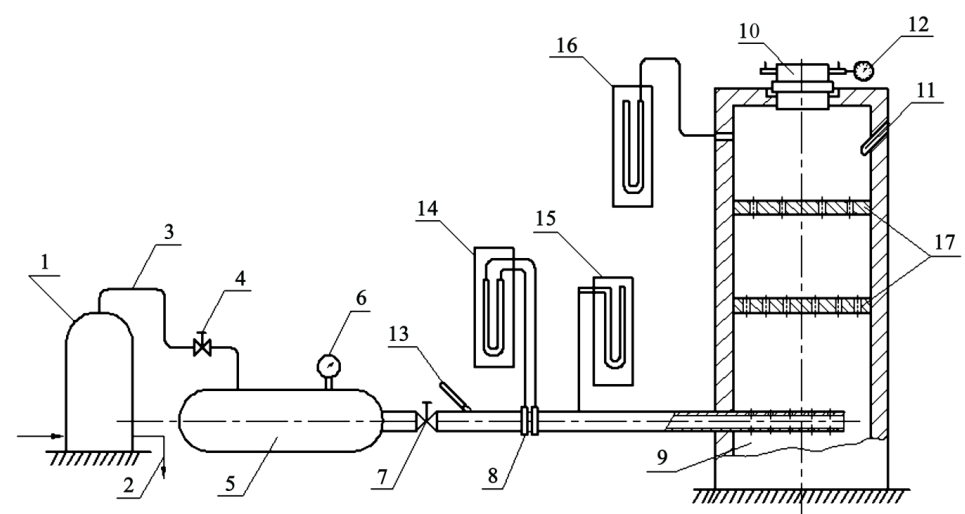

Fig. 1. Scheme of the static valve purge stand: 1 - separator; 2 - fluid in the drainage line; 3 - air line; 4 - valve; 5 - tank; 6 - model pressure gauge; 7 - control valve; 8 - expendable diaphragm; 9 - purge chamber; 10 - socket for installing the valve; 11 - pocket for installing a mercury thermometer; 12 - indicator with a clockwork; 13 - mercury thermometer;

14, 15, 16 - V-shaped pressure gauges; 17 - partitions with drilled holes

Air from the high pressure air line enters the separator 1 and from there through the air line 3 through the valves 4 enters the stilling tank 5, where the standard pressure gauge 6 is installed and then through the control valve 7, the flow diaphragm 8 enters the purge chamber 9. This chamber is a cylinder with two partitions 17 having holes. In the upper part of the chamber there is a socket for installing a valve 10, which is attached to the chamber by a pressure flange. When air is supplied to the purge chamber, the air pressure in the stilling vessel is measured with a standard pressure gauge 6 , the temperature is measured with a mer- 
cury thermometer 13 in front of the diaphragm, and the pressure drop across the V-shaped gauge 14, the pressure behind the diaphragm and in front of the valve are also measured with V-shaped gauges 15 and 16 The air temperature in front of the valve is measured by a mercury thermometer 11. The pipeline section with the flow diaphragm meets the requirements of the Rules for measuring the flow rate of liquids, gases and vapors with standard diaphragms and nozzles [10]. A hole with a diameter of $2 \mathrm{~mm}$ is drilled in the upper part of the valve, into which a needle is inserted, connected to the indicator of the clock mechanism 12, fixing the height of the valve plate with an accuracy of $0.01 \mathrm{~mm}$.

For this experimental stand, the following methodology is adopted for the determination and calculation of some basic parameters characterizing the efficiency of direct-flow valves:

1. The value of the equivalent area was found from the equality of the volumetric flow rate of gas flowing through the diaphragm 8 of the purge chamber 9 and through the valve 10 according to the formula:

$$
\begin{aligned}
& Q_{d}=k \alpha_{d} \varepsilon_{d} F_{d} \sqrt{\frac{\Delta P_{d}}{\rho}}, \\
& Q_{v}=k \alpha_{v} \varepsilon_{v} F_{v} \sqrt{\frac{\Delta P_{v}}{\rho}} .
\end{aligned}
$$

At constant values and the same temperature of the flowing gas, the equivalent valve area is determined by the dependence:

$$
\Phi_{e q}=\alpha_{v} F_{v}=\frac{\alpha_{d} \varepsilon_{d} F_{d}}{\varepsilon_{v}} \sqrt{\frac{\Delta P_{d}\left(P_{d}+B\right)}{\Delta P_{v}\left(P_{v}+B\right)}},
$$

where $k$ - correction factor for the thermal expansion of the material of the diaphragm; $F_{v}-$ area of the valve socket; $\alpha_{v}-$ valve flow coefficient; $\Delta P_{d}$ and $\Delta P_{v}$ - pressure drop in front of the diaphragm and valve, respectively; $B$ - barometric pressure; $\alpha_{d}$ - flow rate through the diaphragm; $\varepsilon$ - coefficient taking into account the expansion of the medium when flowing through the diaphragm is determined by the formula:

$$
\varepsilon=1-0,3 \frac{P_{1}-P_{2}}{P_{1}}
$$

where $P_{1}$ and $P_{2}$ - pressure at the inlet and outlet of the valve (or diaphragm), respectively.

2. The volumetric flow rate of air passing through the diaphragm and through the valve according to the "Rule for measuring the flow rate of liquids, gases and vapors with standard diaphragms and nozzles" [10] is determined by the formula:

$$
Q=0,01252 \alpha_{d} \varepsilon_{d} d^{2} \sqrt{\frac{\Delta P_{d}}{\rho}},
$$

where $d$-diaphragm diameter; $D$ - inner diameter of the pipeline in front of the diaphragm; $\varepsilon_{d}$ - correction factor for the expansion of the measured medium; $\rho$ - air density in front of the diaphragm, determined by the formula:

$$
\rho=\gamma \frac{\left(B+\Delta P_{d}\right) T_{o}}{\Delta P_{v} T},
$$

where $T_{o}$ - gas temperature after the diaphragm; $T$ - gas temperature in front of the diaphragm.

3. The gas velocity in the valve is determined by the ratio: 


$$
C_{v}=\frac{Q_{v}}{F_{s}}
$$

where $Q_{v}$ - current volumetric flow rate through the valve; $F_{C}$ - passage area in the valve seat.

The cross-sectional area of the valve seat is selected for the defining cross section, since this geometric parameter remains constant at any flow rate of gas flowing through the valve, and this parameter is set by the manufacturer.

4. The flow rate of the valve seat is determined from the expression:

$$
\alpha_{v}=\frac{\Phi_{e q}}{F_{s}} .
$$

5. The coefficient of valve resistance is determined from the ratio:

$$
\Delta P_{v}=\xi \frac{C_{v}^{2}}{2 g} \rho
$$

where $\Delta P_{v}-$ current value of the pressure drop across the valve.

6. The Reynolds number Re, referred to the valve bore diameter, is determined by the following relation:

$$
\operatorname{Re}=\frac{C_{v} \cdot d_{v}}{v}
$$

where $d_{v}$ - valve bore diameter; $v=\frac{\mu}{\rho}$ - kinematic viscosity; $\rho$ - gas density in front of the diaphragm; $\mu$-dynamic viscosity of the medium under operating conditions; at $\mu=1,8 \cdot 10^{-6} \mathrm{~kg} / \mathrm{m} \cdot \mathrm{s}$, $t=10{ }^{\circ} \mathrm{C}$ and $P=0.10382 \mathrm{MPa}$, the kinematic viscosity will be:

$$
v=\frac{1,8 \cdot 10^{-6} \cdot 9,81}{\rho}=\frac{1,77 \cdot 10^{-7}}{\rho} \mathrm{m}^{2} / \mathrm{s} .
$$

Then

$$
\operatorname{Re}=\frac{C_{v} \cdot \rho \cdot d_{v}}{1,77 \cdot 10^{-7}}
$$

7. The pressure coefficient of the gas flow on the plate is determined by the formula:

$$
C_{w}=\frac{2 P_{2}}{f_{p l} \cdot \rho \cdot C_{v}^{2}},
$$

where $P_{2}$ - elastic force of the plate is determined by the formula:

$$
P_{2}=6 \frac{D h}{H_{0}^{3}},
$$

where $D$ - stiffness of the plate, which is determined by the formula:

$$
D=\frac{E B \delta^{3}}{22\left(1-\mu^{2}\right)} \text {. }
$$

The tightness of the valves is determined according to the recommendations of [11] in the purge chamber, with the difference that the valve is installed in such a way that the gas flow from 
the chamber pressed the plates to the seats, that is, the upstream side of the valve. The purge chamber filled with gas to a pressure of $0.5-0.6 \mathrm{MPa}$ is disconnected from the compressed gas network and, as a result of leaks through the valves, the pressure in the chamber gradually decreases. At the moment of reaching an overpressure of $0.4 \mathrm{MPa}$ in the chamber, the stopwatch is turned on, and at an overpressure of $0.2 \mathrm{MPa}$, it is turned off.

The valve density criterion is calculated by the formula:

$$
\tau=\tau_{1} \frac{V}{V_{1}} \sqrt{\frac{T_{1}}{T}},
$$

where $\tau_{1}$ - time of excess pressure drop in the purge chamber from 0.4 to $0.2 \mathrm{MPa} ; V$ - volume of the capacity of the test vessel in accordance with GOST26-12-384-72 (0.064 $\left.\mathrm{m}^{3}\right) ; V_{1}-$ actual volume of the purge chamber capacity, $\mathrm{m}^{3} ; T$ - temperature of compressed air, $\mathrm{K}$ ( $\left.T=293.15 \mathrm{~K}\right) ; T_{1}$-actual temperature of the compressed gas, $\mathrm{K}$.

Valve purges confirmed that in the zone corresponding to the expiration mode under the actual valve operating conditions $\left(\operatorname{Re}>10^{8}\right)$, the value of the flow coefficient, as well as the equivalent area, does not depend on the Reynolds number, i.e., a self-similar expiration mode occurs, the achievement of which is necessary to ensure the similarity of the flow conditions in real ones.

Currently, there is no pre-compiled methodology for determining the cause and possibility of valve leakage. However, during the operation of the mining and metallurgical complex, every 2 hours at the gas-compressor station, the technological parameters of associated petroleum gas are practically measured from the suction and discharge sides, and if pressure and temperature are exceeded, operating personnel stop the oil and gas complex and perform a thorough inspection. Having discovered defects in the valve plate or seat, as well as accumulation of soot in the plate, they carry out works to eliminate defects, the results are entered in the technical journal about defects.

Valves were tested with different leakage criteria, during which the leakage through the valve was determined depending on the pressure drop across the valve.

Based on the measurement results, a graph is constructed of the dependence of air leaks through the valve on the magnitude of the pressure increase in the compressor cylinder of the first stage of the mining and metallurgical complex (Fig. 2).

When constructing the diagrams, the compression process was adopted polytropic, and the polytropic index $n_{\text {com }}=1.4$ was adopted, regardless of the degree of pressure increase. The polytropic exponent of the expansion process was accepted differently. According to the recommendations of M. Frenkel with a degree of pressure increase $\varepsilon=2,4,6$, respectively $n_{\text {exp }}=1.5 ; 1.4 ; 1,5$.

When considering gas leaks simultaneously through the discharge and suction valves of the compressor cylinder of the first stage, the value of the leakage criterion of both valves was assumed to be the same.

The graphs show the dependence of gas leaks for one cycle on the pressure degree increase through valves with different values of the tightness criterion.

Each graph shows gas leaks through the suction valve, discharge valve, and leaks through both valves simultaneously.

These graphs are built for a double-acting first stage compressor cylinder with a diameter of $320 \mathrm{~mm}$ of a gas motor compressor, which is widely used in gas-lift operation of oil wells.

The graphs indicate that as the valve tightness increases, gas leakage from the cylinder of the 1st stage is significantly reduced.

With a valve tightness criterion of $35 \mathrm{~s}$, the productivity loss due to gas leakage through the valve plates is $0.6 \%$ at $\varepsilon=2$ and $1.2 \%$ at $\varepsilon=6$. In this connection, the criterion for the tightness of pressure valves should be greater than suction valves.

An experimental study of the tightness of direct-flow valves revealed that gas leakage through the discharge valve plates, depending on the operating mode, is 1.5-2 times higher than through the suction valve. The maximum value of the valve tightness criterion is established at $35 \mathrm{~s}$, at which gas leakages do not exceed $0.6 \%$. 


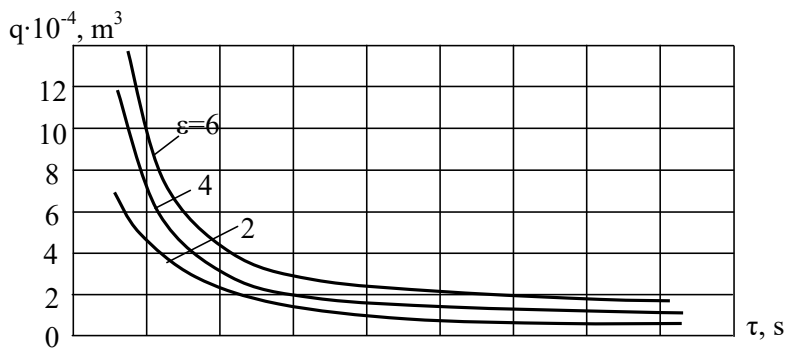

$a$

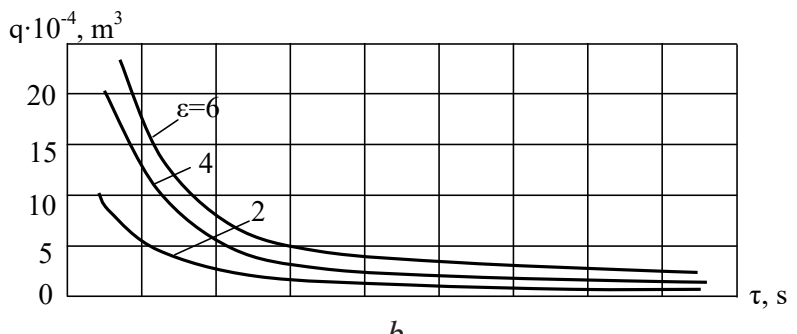

$b$

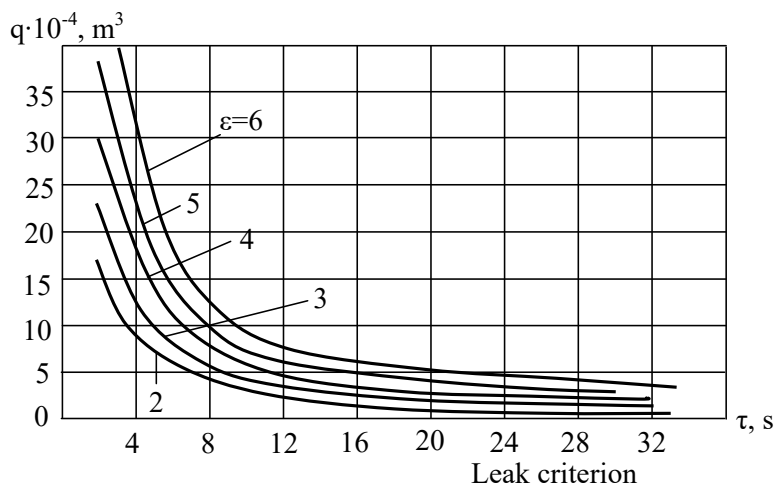

$c$

Fig. 2. The dependence of the amount of gas leakage (\%) through valve leaks in the compressor cylinder $Ø 320$ mm GMC: $a$ - Suction valve; $b$ - Discharge valve; $c$ - Total leaks

In addition, the practical work carried out in the field directly on the existing GCS allows to study certain properties of the valves of compressor cylinders. Therefore, some control tests carried out allows to determine the tightness of the valves.

Directly on the existing GCS, experimental studies have been conducted. On the compressors of two GCS, valves were installed without purging in the purge chamber, and on the compressors of two other GCS, valves are installed after purging. After installing these valves on the compressor cylinders of the first stage of the GMC, they are put into operation in the gas lift system and measured all the parameters of the valves in the same mode of compression of associated petroleum gas. Parameters are recorded approximately every 2 hours to determine tightness.

The operation of the valves shows that during operation their performance increased by about 1.5-2 times. Practical studies have shown that valves without purging worked for 200-250 hours, and valves with purging work for 400-450 hours. These numbers of the valve density criterion correspond to the average data obtained from the leak test results of more than 50 valves.

In the process of experimental studies, random damages to direct-flow valves also occurred, for example, destruction associated with the accidental ingress of solid particles with a gas stream, as well as due to worn piston rings, and destruction at the places of cutting the plate fillet. 


\section{Discussion of the results of experimental studies}

A comprehensive practical study of the operation of piston compressor valves operating in gas lift systems and associated petroleum gas transport is aimed at increasing their tightness, efficiency, maintainability and safe operation $[12,13]$.

Experimental studies were carried out according to the recommendations indicated in $[14,15]$. The basic principles of the field test direction are in verification of the results of a practical study of the operation of valves of the compressor cylinders of the 1st stage, which affect the performance, specific power consumption and safe operation of reciprocating compressor units.

Field tests of the operation of the valves of the compressor cylinders of the 1st stage are an extremely complex process, which allows improving the work of checking, purging, cleaning the valves installed on the compressor cylinders of the 1st stage to increase the GMC efficiency. It follows that the full-scale tests of the operation of valves at the gas compressor station determine the overall GMC performance and the actual technological parameters of the general gas compressor station associated with the technological parameters of the gas-lift oil production system. The purpose of field tests is, as a rule, a practical study of the working process of the valve installed on the 1st stage compressor cylinder and ways to minimize gas leakage through the valve during GMC safe operation, increasing the efficiency of the 1st stage compressor cylinders and efficiency, reducing irrational leakage losses due to leakage of the valve plate. Moreover, before testing the operation of the valves, the completeness of the 1st stage compressor cylinders, the systems for measuring technological parameters and the cooling system of compressor cylinders were carefully checked. Checks were carried out in a sufficient number of values in the instrument reading area that corresponded to the test conditions of the compressor cylinder of the GMC first stage. All characteristics of devices and valve parts used in the testing process were recorded in the journal, especially for valve parts, pressure gauges and thermometers. At the same time as the selection of valves and devices, spare gasket materials, tools and copper tubes were also selected. Before testing the operation of the valves of the compressor cylinders of the first stage, the GMC operation was checked. If malfunctions were detected in the operation of the compressor cylinder or its individual components, after finding out the reasons, it was eliminated and a repeated check was carried out before the normal operation of the cylinders. The essence of the method of step-by-step verification was that the poor operation of the complex design of the compressor cylinder of the 1 st stage, interconnected with other GMC components and systems, did not lead to the failure of other components and systems.

Maintenance work was carried out until the complete commissioning of the entire GMC as a whole. After that, the GMC readiness for the full-scale test during its operation in the gas lift system was agreed with the engineering and technical staff of the GCS machine room and then continued practical tests of the valves.

\section{Conclusions}

Thus, field tests of valve operation show that in valves, when there is a violation of the tightness of the plates, this process is very reflected in the technical and economic indicators of the gas condensate complex, the efficiency of the compressor cylinders decreases, and the emergency conditions increase.

To confirm the feasibility of checking the valve tightness, for each gas-lift compressor station, special equipment is proposed on which the valve tightness is checked. According to the developed method, the parameter determining the tightness and efficiency of the valve is considered the time of pressure drop in the tank from 0.4 to $0.2 \mathrm{MPa}$. The maximum value of the valve tightness criterion is established at $35 \mathrm{~s}$, at which gas leakages do not exceed $0.6 \%$. Checking the tightness of the valve plates at the purge installation makes it possible to increase the efficiency and safety of valve operation by $1.5-2$ times.

\section{References}

[1] Aliev, V. I. (2007). Nauchnye osnovy povysheniya ehffektivnosti raboty gazomotokompressorov v sistemah gazlifta i transporta gaza morskih mestorozhdeniy Azerbaydzhana. Baku, 317. 
[2] Lieberman, N. (2019). How Compressors Work. Understanding Process Equipment for Operators and Engineers, 241-250. doi: https://doi.org/10.1016/b978-0-12-816161-6.00031-x

[3] De Coning, P., Swinley, J. (2019). Valves. A Practical Guide to Gas Analysis by Gas Chromatography, 133-163. doi: https:// doi.org/10.1016/b978-0-12-818888-0.00005-x

[4] Townsend, J., Badar, M. A., Szekerces, J. (2016). Updating temperature monitoring on reciprocating compressor connecting rods to improve reliability. Engineering Science and Technology, an International Journal, 19 (1), 566-573. doi: https://doi.org/ 10.1016/j.jestch.2015.09.012

[5] Pont, A., López, J., Rigola, J., Pérez-Segarra, C. D. (2017). Numerical dynamic analysis of reciprocating compressor mechanism. Parametric studies for optimization purposes. Tribology International, 105, 1-14. doi: https://oi.org/10.1016/j.triboint. 2016.06.019

[6] Sharma, V., Parey, A. (2019). Performance evaluation of decomposition methods to diagnose leakage in a reciprocating compressor under limited speed variation. Mechanical Systems and Signal Processing, 125, 275-287. doi: https://doi.org/10.1016/ j.ymssp.2018.07.029

[7] Loukopoulos, P., Zolkiewski, G., Bennett, I., Sampath, S., Pilidis, P., Duan, F. et. al. (2019). Reciprocating compressor prognostics of an instantaneous failure mode utilising temperature only measurements. Applied Acoustics, 147, 77-86. doi: https:// doi.org/10.1016/j.apacoust.2017.12.003

[8] Qi, G., Zhu, Z., Erqinhu, K., Chen, Y., Chai, Y., Sun, J. (2018). Fault-diagnosis for reciprocating compressors using big data and machine learning. Simulation Modelling Practice and Theory, 80, 104-127. doi: https://doi.org/10.1016/j.simpat.2017.10.005

[9] Reis, M. N. E., Hanriot, S. (2017). Incompressible pulsating flow for low Reynolds numbers in orifice plates. Flow Measurement and Instrumentation, 54, 146-157. doi: https://doi.org/10.1016/j.flowmeasinst.2017.01.001

[10] Pravila 28-64 izmereniya rashoda zhidkostey gazov i parov standartnymi diafragmami i soplami (1978). Moscow, 151.

[11] Plastinin, P. I. (2008). Porshnevye kompressory. Vol. 2. Moscow: Kolos S, 711.

[12] Kudźma, Z., Stosiak, M. (2015). Studies of flow and cavitation in hydraulic lift valve. Archives of Civil and Mechanical Engineering, 15 (4), 951-961. doi: https://doi.org/10.1016/j.acme.2015.05.003

[13] Loukopoulos, P., Zolkiewski, G., Bennett, I., Sampath, S., Pilidis, P., Li, X., Mba, D. (2019). Abrupt fault remaining useful life estimation using measurements from a reciprocating compressor valve failure. Mechanical Systems and Signal Processing, 121, 359-372. doi: https://doi.org/10.1016/j.ymssp.2018.09.033

[14] Mirzadzhanzade, A. H., Stepanova, G. S. (1977). Matematicheskaya teoriya ehksperimenta v dobyche nefti i gaza. Moscow: «Nedra», 228.

[15] Nalimov, V. V., Chernova, N. A. (1965). Statisticheskie metody planirovaniya ehkstremal'nyh ehksperimentov. Moscow: «Nauka», 345 . 